\title{
Endogenic intoxication as a reflection of metabolic disorders in cows and possibilities of its pharmacological correction
}

\author{
Marina P. Semenenko ${ }^{1 *}$, Tatyana A. Sakhno ${ }^{1}$, Elena $V$. Kuzminova $^{1}$, and Aleksey V. Savinkov ${ }^{2}$ \\ ${ }^{1}$ Krasnodar Research Center for Animal Husbandry and Veterinary Medicine, Krasnodar, 350055, Russia \\ ${ }^{2}$ Samara State Agrarian University, 446442, Kinel, Samara Region, Russia
}

\begin{abstract}
The article presents the results of studies of medium mass molecules concentration in the blood serum of cows with different physiological conditions and correction of this state with livazen, which has an antioxidant and lipotropic effect on liver cells. It was determined that the use of livazen contributed to a decrease in cholesterol in the group of pregnant cows by $21.0 \%$ and in the group of fresh cows by $15.9 \%$, with a decrease in the concentration of average molecular peptides by 4.0-11.8\% depending on the length of exposure.
\end{abstract}

\section{Introduction}

The modern development of dairy farming is based on the further increase in milk production through the introduction of modern technologies for feeding and keeping animals, optimizing herd reproduction and increasing the genetic potential of dairy cattle.

However, imbalance of feeding diets, violation of the maintenance technology, increased intensity of exposure to harmful environmental factors, as well as low adaptive potential of the animals themselves, lead to intense work of organs and systems of highly productive cows. It results in appearance of a wide range of physiological abnormalities leading to the development of a common metabolic pathology, manifested in the form of a metabolic syndrome [1].

As a rule, such a common metabolic pathology is distinguished by a long latent course and the absence of pathognomonic signs, while being a serious prerequisite for the occurrence of many diseases. In the process of proteolysis in the damaged cells of the body, the socalled medium mass molecules (MMM) or medium molecular peptides (MMP) are formed - biologically active substances of peptide nature, as well as derivatives of oligosols and glucuronic acid, which have a different origin.

Their chemical composition is heterogeneous and unites a heterogeneous group of substances. It includes peptides, glycopeptides, nucleopeptides, endorphins, amino sugar, polyamines, polyhydric alcohols, some humoral regulators - insulin, glucagon, some vitamins, nucleotides, oligosaccharides, derivatives of glucuronic acids and others [2-5].

Medium molecular peptides are the main substrate responsible for the occurrence of the pathological effects of the endogenous intoxication in various diseases. That is, the disruption of the functioning of the protease and antiprotease systems as a result of the activation of proteolysis leads to accumulation of a large number of protein degradation products with a molecular weight of 300-5000 Da.

A feature of MMM is a pronounced high biological activity. Their accumulation is not only a marker of endointoxication, but can also aggravate the course of the pathological process in the future, affecting the vital functions of all systems and organs. Medium mass molecules have neurotoxic activity, inhibit protein biosynthesis, uncouple oxidation and phosphorylation processes, and disrupt the regulation mechanisms of adenyl nucleotide synthesis. They change ion transport through membranes, erythropoiesis, phagocytosis, microcirculation $[6,7]$.

In veterinary medicine, the definition of medium mass molecules practically has not been studied, although their timely detection in the blood of animals can provide the great diagnostic value and serve as a prognostic criterion for assessing the severity of the pathological process. They may also serve as a marker of the effectiveness of the treatment and prognosis of the disease [8].

The obtained results on the registration of low and medium molecular weight substances allow us to prescribe intensive therapy in a timely manner aimed at eliminating endotoxins from the body using the differentiated use of pharmacological preparations, which is a pathogenetic justification for the inclusion of efferent metabolic correction methods in animals with metabolic disorders and endotoxicosis.

Moreover, since the development of the general metabolic pathology in the body is primarily associated with the impaired functional activity of liver cells, which, as the main organ of metabolism, is directly involved in a variety of biochemical reactions, pharmacocorrection must be carried out using substances

Corresponding author: sever291@mail.ru 
that have a directed effect on hepatocytes hepatoprotectors [9].

Therefore, the aim of our study was to assess the level of medium mass molecules in the blood of pregnant and fresh cows before and after the use of the livazen preparation.

\section{Materials and Methods}

Livazen is an injectable preparation, appearing as a transparent solution, odourless, with slightly oily consistency. The mechanism of action of the diisopropylammonium dichloroacetate included in the preparation consists of its antioxidant and membranestabilizing effect. It inhibits the processes of lipid peroxidation, increases the activity of superoxide oxidase, the ratio of lipid to protein, reduces the viscosity of the membrane, and increases its fluidity. It modulates the activity of membrane-bound enzymes, receptor complexes, which enhances their binding ability; helps preserve the structural and functional organization of biomembranes. Diisopropylammonium dichloroacetate exerts a lipotropic effect, favourably affects the antitoxic and pigment function of liver, and increases resistance to hypoxia and various types of intoxications [10].

To study the endotoxic properties of the livazen preparation, four groups of Holstein cows were formed 10 animals in each - two experimental and two control groups. The first experimental group consisted of animals that were in the fifth month of pregnancy, the second experimental group consisted of fresh cows.
The animals of the experimental groups received livazen intramuscularly at a prophylactic dose $(2 \mathrm{ml})$ for 14 days. The control groups of animals used saline method of administration in the same doses.

Evaluation of the results was carried out with respect to the dynamics of cholesterol and medium mass molecules, for which all cows, selected in the experiment, were sampled at the beginning of the experiment and at the end of it. It is known that during pregnancy there are significant changes in lipid metabolism, therefore, the study of cholesterol is an important biochemical indicator for assessing the level of endogenous intoxication of the body.

Studies of cholesterol were carried out on a Vitalab Flexor Junior automated biochemical analyzer (Netherlands) using reagents from ELITech Clinical Systems (France) and Analyticon biotechnologies AG (Germany). The level of endogenous intoxication was determined according to the method of N.I. Gabrielyan and V.I. Lipatova (1984) by quantitatively evaluating the content of MMM at a wavelength $(\lambda)$ of 254 and $280 \mathrm{~nm}$.

The digital data obtained in the experiments were processed using the statistical software package Statistica 6.0. Data are presented as mean X and standard error of mean $\mathrm{m}$. The significance of differences between the series was determined using Student's tcriterion.

\section{Results}

An analysis of the results of the biochemical study of the blood serum found out that the use livazen in cows had a significant impact on cholesterol and MMM (Table 1).

Table 1. The influence of livazen on cholesterol and medium molecular peptides in the body of cows $(M \pm m ; n=5)$

\begin{tabular}{|c|c|c|c|c|c|c|c|c|}
\hline \multirow{3}{*}{ Indicators } & \multicolumn{4}{|c|}{ Pregnant cows } & \multicolumn{4}{c|}{ Fresh cows } \\
\cline { 2 - 9 } & \multicolumn{1}{|c|}{ Beginning of the experiment } & \multicolumn{2}{|c|}{ End of the experiment } & \multicolumn{2}{|c|}{ Beginning of the experiment } & \multicolumn{2}{c|}{ End of the experiment } \\
\cline { 2 - 9 } & Experimental & Control & Experimental & Control & Experimental & Control & Experimental & Control \\
\hline $\begin{array}{c}\text { Cholesterol, } \\
\mathrm{mmol} / \mathrm{ml}\end{array}$ & $6.3 \pm 0.2$ & $4.9 \pm 0.2$ & $5.0 \pm 0.29$ & $5.7 \pm 0.05$ & $5.6 \pm 0.35$ & $4.1 \pm 0.2$ & $4.7 \pm 0.1$ & $4.6 \pm 0.30$ \\
\hline $\mathrm{MMM}_{254, \mathrm{AU}}$ & $0.20 \pm 0.01^{* *}$ & $0.21 \pm 0.01$ & $0.17 \pm 0.01^{* *}$ & $0.24 \pm 0.01$ & $0.27 \pm 0.01$ & $0.25 \pm 0.02$ & $0.25 \pm 0.01$ & $0.28 \pm 0.01$ \\
\hline $\mathrm{MMM}_{280} \mathrm{AU}$ & $0.17 \pm 0.01^{* *}$ & $0.16 \pm 0.01$ & $0.15 \pm 0.01^{* *}$ & $0.22 \pm 0.01$ & $0.23 \pm 0.01$ & $0.22 \pm 0.02$ & $0.23 \pm 0.02$ & $0.29 \pm 0.02$ \\
\hline
\end{tabular}

Note: $* *$ - significance level $\mathrm{p} \leq 0.01$

So, the concentration of cholesterol in the first experimental group decreased by $21.0 \%$ compared to the background values; in the second experimental group it decreased by $15.9 \%$, respectively. Whereas, in the control groups, on the contrary, an increase in this indicator by 16.3 and $12.2 \%$ was noted.

Cholesterol is an organic compound contained in the cell plasma membrane of all cells of a living organism and plays the role of a bilayer modifier, giving certain rigidity by increasing the density of the "packing" of phospholipid molecules.

That is, cholesterol, in fact, is a stabilizer of fluidity of the plasma membrane. With the development of metabolic disorders in the animal organism, primarily suffer the cell membranes, especially their lipid component.

Damage to the lipid component of the epithelial membranes leads to disruption of cellular homeostasis and activation of free radical oxidation processes that accompany various pathological conditions.

In this regard, the effectiveness of therapy largely depends on the degree of protection of the structure and function of cell membranes and the limitation of the activity of free radical oxidation processes, including the help of preparations [11]. The use of livazen that has a lipotropic effect helped to reduce the degree of fatty infiltration and to improve lipid metabolism in the liver hepatocytes of experimental cows.

The indicator of medium mass molecules at the beginning of the experiment in the first experimental group decreased, depending on the duration of exposure, by 15.0 and $11.8 \%(\mathrm{p} \leq 0.01)$. It objectively indicates a decrease in the intensity of catabolic processes, which are the main a source of medium molecular endogenous toxins, as well as shows an improvement in the functioning of integral (detoxification) systems of the 
body, reflecting the dynamics of metabolic shifts towards improvement (Fig. 1).

The level of medium mass molecules in the experimental group of fresh cows with an exposure length of 254 AU decreased by $7.4 \%$, and remained unchanged at a wavelength of 280 AU (Fig. 2).

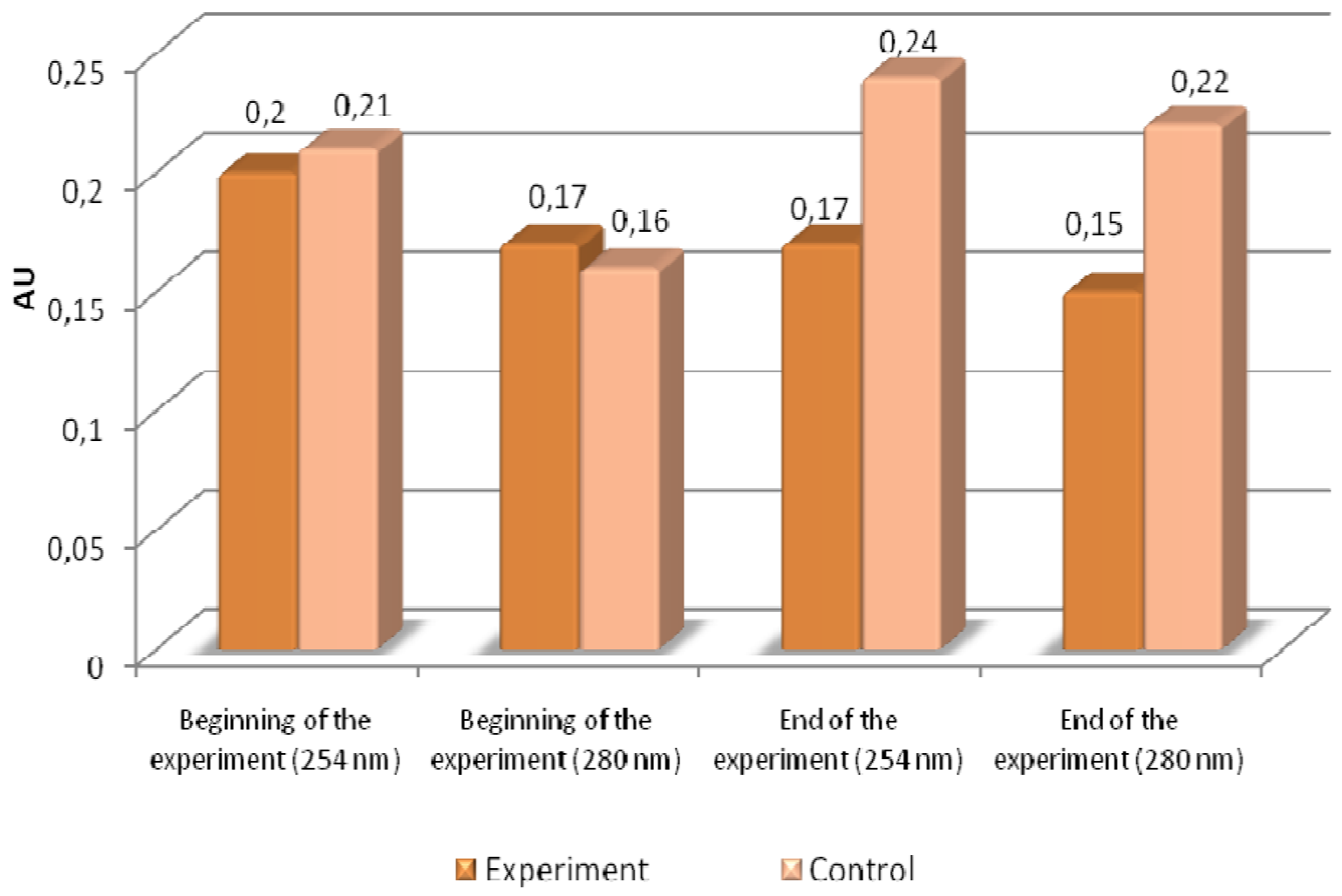

Fig. 1. The influence of livazen on the level of medium mass molecules in pregnant cows

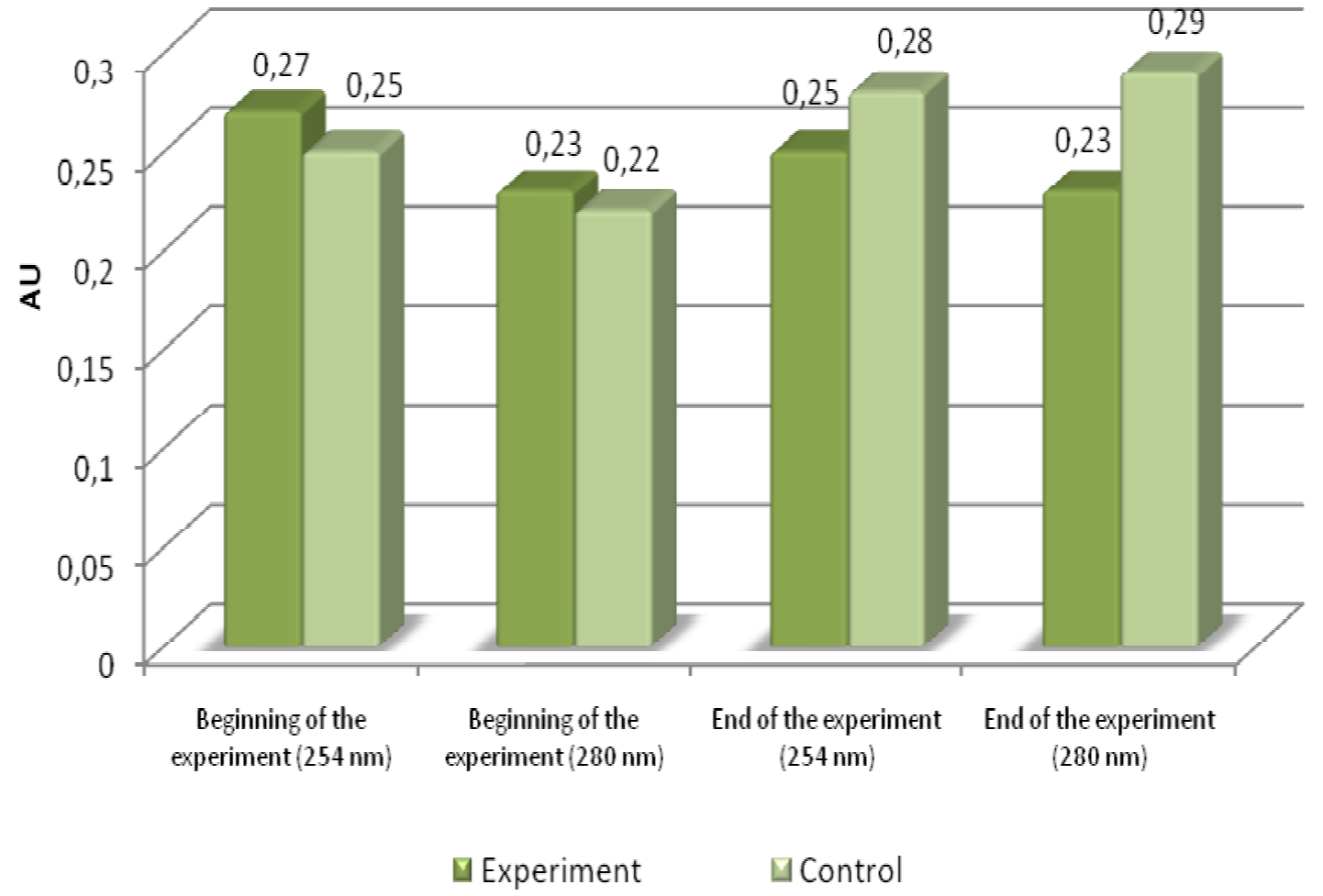

Fig. 2. The influence of livazen on the level of medium mass molecules in fresh cows 
It should be noted that at a wavelength of $\mathrm{MMM}_{254} \mathrm{~nm}$, hydrophobic toxins can be found that are highly similar to biological structures, located in plasma and in an almost completely bound state in the form of complexes with low-density albumin or lipoproteins.

At the same time, the average concentration of MMM at a wavelength of $280 \mathrm{~nm}$ contains aromatic chromophores, which are the remains of aromatic (tryptophan, tyrosine, phenylalanine), heterocyclic (histidine) and sulfur-containing (cystine) amino acids.

In this case, we can say that in pregnant cows there was an imbalance between the individual components of the pool of medium mass molecules in the direction of an increase in substances formed as a result of catabolism and which are the products of cell decay, xenobiotics, and substances of microbial nature. Whereas, in this physiological period the destruction of amino acids was not pronounced. Perhaps the use of the preparation stabilized the flow of biochemical reactions in the body, ensuring its normal functioning.

The level of MMM in the control groups increased regardless of the physiological state of the animals.

In pregnant cows, by the end of the experimental period, the number of medium molecular peptides increased by $14.3 \%$ (with an exposure length of 254 $\mathrm{AU}$ ) and by $37.5 \%$ (with a wavelength of $280 \mathrm{AU}$ ). After calving, endotoxemia in control cows was not pronounced. The differences between the background and the final indicators were 12.0 and $26.1 \%$, respectively.

The difference between the MMM values in pregnant cows of the experimental and control groups by the end of the research was 29.2 and $31.8 \%$; in fresh cows it was 10.7 and $20.7 \%$, respectively.

\section{Conclusion}

Thus, the spectrum of pharmacological and prophylactic activity of the livazen preparation is manifested by a decrease in the level of medium mass molecules and, above all, peptides with a lower molecular weight, thereby leading to a decrease in the severity of endogenous ("metabolic") toxicosis in cattle. In this case, the most pronounced effect of the preparation is observed in animals during pregnancy, when the body accumulates toxic substances of exogenous and endogenous nature, which are the impetus for the development of many pathological conditions.

In the postpartum period its regression is noted, therefore, the level of endogenous intoxication can decrease due to internal processes of the body itself. Consequently, the level of the increase in the concentration of MMM, as well as their distribution between biological media depends on the physiological state of the animals.

Data analysis allows us to conclude that there is the predominance of the catabolic pool in pregnant cows over the anabolic pool and the accumulation of medium mass molecules in blood resulting from the destruction of hepatocyte cell membranes. Their maximum decrease after treatment with livazen confirms its hepatoprotective effect, aimed at restoring the integrity of the membrane of the liver cells.

\section{References}

1. E.V. Kuzminova, M.P. Semenenko, S.I. Kononenko, N.N. Zabashta, Pharmacoprophylacxis system of liver diseases in highly productive daily cattle, Bulletin of Timiryazev Agricult.1 Acad., 4, 121-132 (2019)

2. L.M. Obukhova, N.A. Andriyanova, Determination of substances of low and medium molecular mass in blood serum as an additional diagnostic criterion for fatal drug poisoning, Forens.-med. Examinat., 6, 37-39 (2014)

3. V.I. Sidel'nikova, A.E. Chemitskiy, M.I. Retsky, Endogenous intoxication and inflammation: reaction sequence and informativity of the markers (review), Agricult. Biol., 50(2), 152-161 (2015)

4. E.V. Karjakina, S.V. Belova, Medium mass molecules as an integral indicator of metabolic disorders (literature review), Clin. Labor. Diagn., 3, 4-8 (2004)

5. L.L. Pan, H.J. Zhang, Z.F. Huang et al., Intrahepatic triglyceride content is independently associated with chronic kidney disease in obese adults: A crosssectional study, Metabol., 64, 1077-1085 (2015)

6. A.Yu. Shitov, Medium mass molecules as an indicator of "hyperboric intoxication" in divers, Clin. Med. Alm., 28, 48-52 (2013)

7. M.Yu. Marzhokhova, M.M. Nagoyeva, M.M. Afashagova, A.R. Marzhokhov, A.A. Shaova, Evaluation of the degree of intoxication and prognosis for the level of the integrated intoxication index for some infectious diseases, Arch. of Internal Med., 2(28), 46-50 (2016)

8. M.P. Semenenko, E.V. Kuzminova, E.V. Tyapkina, A.A. Abramov, K.A. Semenenko, Molecules of Medium Mass as an Integral Indicator of Endogenous Intoxication in the Diagnosis of Hepatopathy and its Effect on Improving the Economic Efficiency of Veterinary Measures in the Field of Dairy Farming, J. of Pharmac. Sci. and Res. (JPSR), 9(9), 1573-1575 (2017)

9. R. Weiskirchen, Hepatoprotective and Anti-fibrotic Agents: It's Time to Take the Next Step, Front. Pharmacol., 6(303), 1-40 (2016)

10. B.P. Strunin, A.T. Gubaidullin, T.V. Pakhomova et al., The structure of the preparation diisopropylammonium dichloroacetate, Bull. of Kazan Technol. Univer., 3, 145-148 (2013)

11. M.I. Balabolkin, E.M. Klebanova, The role of oxidative stress in the pathogenesis of vascular complications, Endocrinol. Probl., 6, 29-34 (2000)

12. E.P. Yakovenko, P.Ya. Grigoryev, Chronic liver diseases: diagnosis and treatment, Russ. Med. J., 11(5), 291 (2003) 